\title{
NUMERICAL ANALYSES FOR THE MATERIAL BIFURCATION IN PLANE SHEET UNDER TENSION
}

\author{
T. HUANG and G. C. LI \\ Institute of Mechanics, Academia Sinica, Beijing 100080, P.R. China
}

\begin{abstract}
The various patterns (shear banding, surface wrinkling and necking) of material bifurcation in plane sheet under tension are investigated in this paper by means of a numerical method. It is found that numerical analysis can provide better ground for searching for the lowest critical loads. The inhomogeneity caused by void damage and the nonuniformity in the stress distribution across sheet thickness are proved to have detrimental effects on the material bifurcation. Nevertheless, material stability can be promoted by any means of depressing void damage or alleviating stress, even locally across the thickness. Besides, the peculiar behaviour of material bifurcation under slight biaxiality state is demonstrated. Copyright (C) 1996 Elsevier Science Ltd
\end{abstract}

\section{INTRODUCTION}

IT IS commonly known that the incipient failure in ductile materials is the occurrence of material bifurcation. Surface wrinkling, shear banding and necking can all act as precursor to eventual fracture. To improve the understanding of their formation mechanism is conducive to developing the capacity for predicting material failure, which is extremely important for the efficient control of the metal forming process.

Employing incompressible plasticity theory, Hill and Hutchinson [1] proposed a localizedmode solution method for analysing the shear-band bifurcation. $\mathrm{Li}$ and $\mathrm{Zhu}$ [2] developed a diffuse-mode analysis, taking into account the effect of plastic dilatancy caused by voiding in ductile materials. However, owing to the limitation in prescribing the bifurcation patterns as pre-requested in this analytical method, it is not always able to obtain the lowest critical value for the different patterns.

Material bifurcations in continuous media are generally sensitive to heterogeneities. Using Gurson's [3] model proposed for voided materials, Yamamoto [4], Ohno and Hutchinson [5] and Becker [6] studied the role of nonuniform distributions of voiding in forming the shear band. Huang [7] investigated the role of nonuniform particle distribution in plastic flow localization.

In this paper, numerical method is utilized to enhance the study of the various patterns (shear banding, surface wrinkling and necking) of material bifurcation in plane sheet under plane-stress tension. As the local distributions of velocity variated at bifurcation can be well discretized by this method, the lowest critical value is able to be searched within a wide scope of variation patterns.

The numerical method is also an exclusive approach to the analysis of the effects caused by the inhomogeneity of void damage and by the nonuniformity of stress distribution across sheet thickness. The effect of having slight tension along the transverse direction is also studied, since pure uniaxial tension is not always available in practical testings. The results obtained in this paper can help understand their influence on material bifurcation and set up theoretical basis for providing means to improve material ductility.

\section{THEORETICAL FORMULATIONS}

Ensuring that the functional

$$
\Pi=\frac{1}{2} \int\left[\frac{\mathrm{D} \tau_{i j}}{\mathrm{D} t} \cdot D_{i j}-\sigma_{i j}\left(2 D_{i k} D_{i k}-V_{k, j} V_{k, j}\right)\right] \mathrm{d} v-\int_{v} F_{i} V_{i} \mathrm{~d} s
$$

takes an extremum is equivalent to satisfying the equilibrium equations together with the boundary conditions for any incremental solution of the boundary-value problem in the practical up-dated 
Lagrangian formulation [8]. In which, $\mathrm{D} \tau_{i j} / \mathrm{D} t$ is the Jaumann rate of Kirchhoff stress, $\sigma_{i j}$ is the Cauchy stress, $\dot{F}_{i}$ denotes the rate of external load and the deformation rate $D_{i j}$ is related to the velocity components $V$, as

$$
D_{i l}=\frac{1}{2}\left(V_{i, j}+V_{j, i}\right)
$$

Under the condition of dead (conservative) loading, it has been demonstrated $[2,9]$ that bifurcation occurs at the point when the second variation

$$
\begin{gathered}
Q=\delta^{2} \Pi \\
=\frac{1}{2} \int\left[\frac{\mathrm{D} \delta \tau_{i j}}{\mathrm{D} t} \delta D_{i j}-\sigma_{i j}\left(2 \delta D_{i k} \delta D_{i k}-\delta V_{k, j} \delta V_{k, j}\right)\right] \mathrm{d} v
\end{gathered}
$$

becomes stationary.

In eqs (1) and (3), we have

$$
\frac{\mathrm{D} \tau_{u}}{\mathrm{D} t}=L_{i j k l} D_{k l}
$$

and

$$
\frac{\mathrm{D} \delta \tau_{y j}}{\mathrm{D} t}=L_{i j k}^{*} \delta D_{k l}
$$

In the case when the "linear comparison solid" model can be applied, then $L_{i j k l}^{*}=L_{i j k l}$. In view of the voiding damage in ductile materials, a dilatational plastic constitutive model was lately developed and justified $[11,12]$ which gives the stiffness tensor as

$$
L_{i j k l}=\frac{E}{1+v}\left[\frac{1}{2}\left(\delta_{i k} \delta_{j l}+\delta_{i} \delta_{j k}\right)+\delta_{i j} \delta_{k l} \frac{v-E / 3 E_{\mathrm{lm}}}{1-2 v+E / E_{\mathrm{lm}}}-\frac{3}{2 \sigma_{\mathrm{e}}^{2}} \frac{S_{i} S_{k l}}{1+2(1+v) E_{\mathrm{te}} / 3 E}\right],
$$

where $E$ and $v$ are Young's modulus and Poisson's ratio, respectively. $\sigma_{\mathrm{e}}$ is the equivalent stress, which is related to the deviatoric stress $S_{i j}$ as $\sigma_{\mathrm{e}}=\left(3 / 2 S_{i j} S_{j i}\right)^{1,2}, \delta_{i j}$ represents the Kronecker delta. $E_{\mathrm{te}}=\left(\mathrm{d} \sigma_{\mathrm{e}} / \mathrm{d} \epsilon_{\mathrm{e}}\right)$ and $E_{\mathrm{tm}}=\left(\mathrm{d} \sigma_{\mathrm{m}} / \mathrm{d} \epsilon_{\mathrm{m}}\right)$ are the plastic tangent moduli along the equivalent stress-strain curve $\left(\sigma_{\mathrm{e}}-\epsilon_{\mathrm{c}}\right)$ and the mean stress-strain curve $\left(\sigma_{\mathrm{m}}-\epsilon_{\mathrm{m}}\right)$ respectively, with the mean stress and mean strain defined as $\sigma_{\mathrm{m}}=1 / 3\left(\sigma_{k k}\right)$ and $\epsilon_{\mathrm{m}}=1 / 3\left(\epsilon_{k k}\right)$.

When the finite-difference method is used for numerical solution, we need to normalize the second variation $Q$ and the components of generalized velocity variation $V_{m}^{(n)}$ by taking

$$
\bar{Q}=\frac{Q}{E L^{2}} \text { and } \bar{V}_{m}=\frac{V_{m}^{(n)}}{L},
$$

where $L$ stands for the characteristic length parameter in the bifurcation pattern, $m(=1,2)$ is the $m^{\text {th }}$ component at each nodal point and $(n)(=1,2, \ldots n)$ denotes the sequence number of the discretized nodes with a total number of $n$. The stationary condition at bifurcation can be implemented by vanishing the partial derivatives of $\bar{Q}$ with respect to $\bar{V}_{m}$. Hence, at each nodal point we have two equations in the form of

$$
\partial \bar{Q} / \partial \bar{V}_{m}=0 \quad m=1,2 \text {. }
$$

\section{MATERIAL BIFURCATION IN PLANE SHEET}

Figure 1(a) schematically demonstrates the surface wrinkling with wave length $L$ formed in plane sheet of thickness $h$. It is commonly understood that for surface wrinkling the geometric parameter $h / L$ is much larger than 1 , as shown by Hutchinson and Tvergaard [13] and Tvergaard [14]. This is to distinguish from the necking pattern where the wave length $L$ should be much larger than the plate thickness $h$ and hence $h / L$ is much smaller than 1 , as stated by Burke and Nix [9], owing to the fact that their numerical values for critical strains decrease with respect to the increase of the slenderness ratio of necking sheet under plane-strain tension. A shear band 
(a)

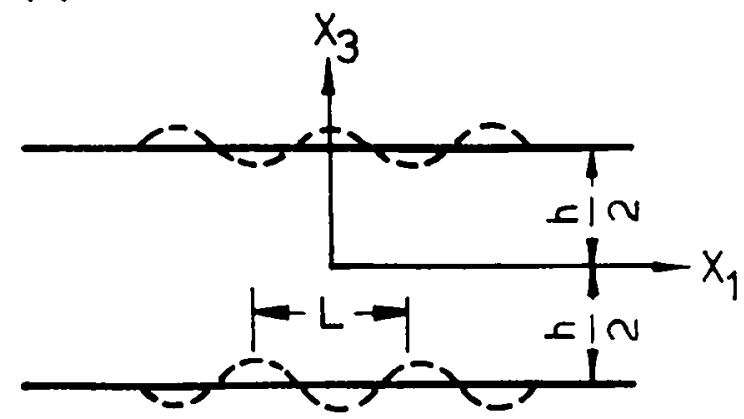

(b)

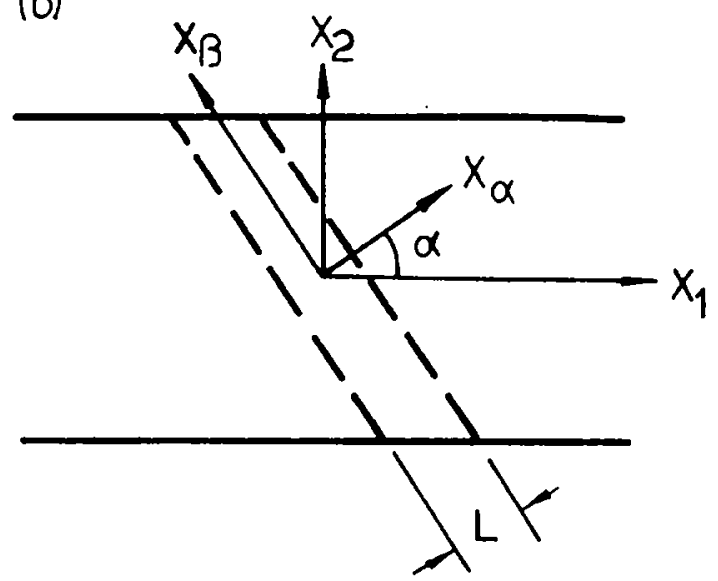

Fig. 1. Schematic demonstration of (a) surface wrinkling and (b) shear banding.

which spans a width of $L$ is shown in Fig. 1(b). Its normal direction is along the axis $x_{x}$ and the tangential direction is parallel to the axis $x_{\beta}$. The angle between $x_{x}$ and $x_{1}$ is nominated as $\alpha$, which can be evaluated in correspondence by approaching the lowest critical loading. Hence, the velocity variations can be represented by taking

$$
\begin{gathered}
\delta V_{1}=\sin \frac{2 \pi x}{L} V_{1}\left(x_{3}\right) \\
\delta V_{3}=\cos \frac{2 \pi x_{1}}{L} V_{2}\left(x_{3}\right),
\end{gathered}
$$

with $\delta V_{2}=0, \delta V_{1,2}=\delta V_{3,2}=0$ for surface wrinkling and

$$
\begin{gathered}
\delta V_{x}=\sin \frac{\pi x_{x}}{L} V_{1}\left(x_{3}\right) \\
\delta V_{\beta}=\sin \frac{\pi x_{z}}{L} V_{2}\left(x_{3}\right),
\end{gathered}
$$

with $\delta V_{3}=0, \delta V_{x, \beta}=\delta V_{\beta, \beta}=0$ for shear banding. In these equations, $V_{1}\left(x_{3}\right)$ and $V_{2}\left(x_{3}\right)$ are the functions used for characterizing the distributions of velocity variations across the sheet thickness $h$. These functions should be discretized by using the finite-difference method.

In the case of surface wrinkling, the boundary conditions that should be satisfied are

$$
\begin{array}{ll}
\delta V_{1}=0, \delta V_{3.1}=0 & \text { at } x_{1}=0 \\
\delta V_{3}=0, \delta V_{1.3}=0 & \text { at } x_{3}=0
\end{array}
$$




$$
\begin{gathered}
\int_{-h \cdot 2}^{h: 2} \delta \dot{T}_{11} \mathrm{~d} x_{3}=0, \int_{-h: 2}^{h \cdot 2} \delta \dot{T}_{13} \mathrm{~d} x_{3}=0 \text { at } x_{1}=L / 2 \\
\delta \dot{T}_{33}=0, \delta \dot{T}_{31}=0 \quad \text { at } x_{3}=h / 2 .
\end{gathered}
$$

Here, $\delta \dot{T}_{11}, \delta \dot{T}_{13}$ and $\delta \dot{T}_{33}$ are components of the variation of nominal stress rate. The boundary conditions for shear banding should change into

$$
\begin{gathered}
\delta V_{x}=0, \delta V_{\beta}=0 \text { at } x_{x}=0 \\
\delta V_{x .3}=0, \delta V_{\beta .3}=0 \text { at } x_{3}=0 \\
\int_{-h \cdot 2}^{h: 2} \delta \dot{T}_{x x} \mathrm{~d} x_{3}=0, \int_{-h: 2}^{h \cdot 2} \delta \dot{T}_{x 3} \mathrm{~d} x_{3}=0 \text { at } x_{x}=L / 2 \\
\delta \dot{T}_{33}=0, \delta \dot{T}_{3 x}=0 \text { at } x_{3}=h / 2,
\end{gathered}
$$

with regard to the $\left(x_{x}, x_{\beta}, x_{3}\right)$ coordinates. For both cases, the pre-bifurcation stress state is taken to have the plane sheet stressed uniaxially, that is to say, only $\sigma_{11}$ is finite while the other stress components are nil.

Taking the material parameters at bifurcation to have $v=0.3, E_{\mathrm{te}} / E=0, \epsilon_{\mathrm{y}}$ (yield strain) $=0.002$ with various values for $E / E_{\mathrm{lm}}(=60,80$ and 100$)$ to simulate the various degree of voiding damage, we can use the numerical procedure stated previously to calculate the critical stresses. Usually 50 finite-difference sections along the half thickness are used for discretization. Its calculation precision has been checked by comparing with that of having 100 sections. The results of both the surface wrinkling and shear banding are listed in Table 1 . The critical uniaxial stress $\sigma_{\mathrm{cr}}$ is being normalized by the yield stress $\sigma_{\mathrm{y}}$. Each stress reaches its lowest value (to three decimal places) at the value of $h / L$ shown in the circular parentheses. The analytical results are based on some previous works $[2,15]$ using trigonometric series (two to three terms) for the functions $V_{1}$ and $V_{2}$ in eqs (9) and (10).

For shear banding, numerical results are equal to analytical results (to three decimal places) due to the precise prescription of bifurcation mode. Since the numerical method has better flexibility in simulating the varieties of velocity forms variated at material bifurcation, it is able to provide lower critical stress as shown in Table 1 for surface wrinkling. Secondly, the results indicated that the different patterns of bifurcation can have critical stresses close to each other. This fact implies that they might be sensitive to the inhomogeneity of materials and the nonuniformity of stress distribution. No necking pattern of bifurcation is found with regard to sheet thickness in pure uniaxial (plane-stress) loading. However, later on it will be shown that slight biaxiality in tension can bring about necking bifurcation at critical stress near to those of shear banding and surface wrinkling.

Figure 2(a) and (b) shows the normalized functions of $V_{2}$ across the half thickness of the sheet. For surface wrinkling, the maximum $V_{2}$, i.e. $\left(V_{2}\right)_{\max }$, occurs at the surface plane $\left(2 x_{3} / h=1\right)$ while conversely the $\left(V_{2}\right)_{\max }$ of shear banding locates at the middle plane $\left(2 x_{3} / h=0\right)$.

\section{THE INHOMOGENEITY OF VOIDING DAMAGE}

According to the experimental report given by Zhu et al. [16] void damage does not have homogeneous distribution across the sheet thickness. Hence, the distribution of void volume fraction $f$, which can be measured, is not uniform across the thickness direction. It is known that

Table 1. The critical stresses $\sigma_{c t} / \sigma_{y}\left(v=0.3, E_{\mathrm{te}} / E=0, \epsilon_{\mathrm{y}}=0.002\right)$

\begin{tabular}{lcccc}
\hline$E / E_{\mathrm{tm}}$ & \multicolumn{2}{c}{$\begin{array}{c}\text { Surface wrinkling } \\
\text { Numerical }\end{array}$} & Analytical & $\begin{array}{c}\text { Shear banding }(h / L=1000) \\
\text { Numerical }\end{array}$ \\
\hline 60 & $2.733(h / L=4-10)$ & $2.750(25-35)$ & 2.750 & 2.750 \\
80 & $2.058(4-12)$ & $2.065(25-35)$ & 2.068 & 2.068 \\
100 & $1.651(3-6)$ & $1.657(25-28)$ & 1.657 & 1.657 \\
\hline
\end{tabular}


the mean strain $\epsilon_{\mathrm{m}}$ caused by voiding is related to the void volume fraction $f$, owing to the fact that

$$
\epsilon_{\mathrm{m}}=\left(\epsilon_{1}+\epsilon_{2}+\epsilon_{3}\right) / 3=f / 3 .
$$

The mean stress $\sigma_{\mathrm{m}}\left(=\sigma_{11} / 3\right.$ under uniaxial stressing) can also be determined by recording the current loading and geometric dimensions. Then the varying values of the tangent modulus $E_{\mathrm{tm}}$, which characterizes plastic dilatancy in eq. (6), can be determined by the relation of

$$
E_{\mathrm{tm}}=\Delta \sigma_{\mathrm{m}} / \Delta \epsilon_{\mathrm{m}} .
$$

One of the main findings given by $\mathrm{Li}$ and $\mathrm{Zhu}$ [2] shows that the rate of porosity development is a controlling factor which favours shear-band bifurcation. This conclusion indicates that $E_{\mathrm{tm}}$ is playing a dominant role in triggering bifurcation. A larger value of $E_{\mathrm{tm}}$ means a slower void growth,
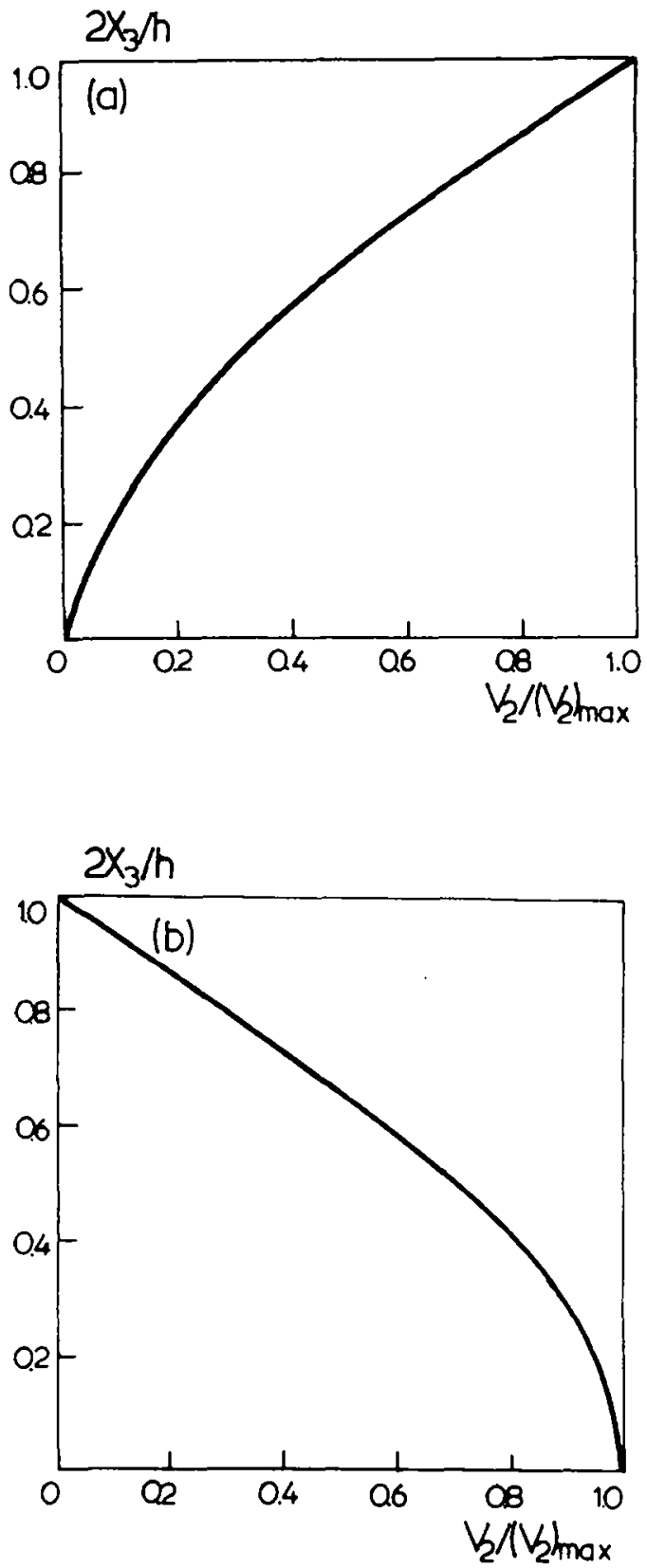

Fig. 2. The distribution of $V_{2}$ across the half thickness of the sheet (a) surface wrinkling, (b) shear banding. 
while a smaller value of $E_{\mathrm{tm}}$ denotes a faster rate of voiding. In this section, we intend to study the effects caused by the inhomogeneity of voiding damage.

Three patterns of inhomogeneity distribution are given in the following to study the difference of their effects on critical stress. The distributions are symmetric with respect to the middle plane at $x_{3}=0$. We use the normalized parameter $E / E_{\mathrm{tm}}$ instead of $E_{\mathrm{tm}}$. We further define

$$
D=\frac{\left(E / E_{\mathrm{lm}}\right)}{\left(E / E_{\mathrm{lm}}\right)_{\mathrm{a}}}, D_{\mathrm{m}}=\frac{\left(E / E_{\mathrm{lm}}\right)_{\mathrm{m}}}{\left(E / E_{\mathrm{tm}}\right)_{\mathrm{a}}},
$$

where the subscript " $\mathrm{m}$ " stands for the maximum (if $D_{\mathrm{m}}>1$ ) or the minimum (if $D_{\mathrm{m}}<1$ ), and the subscript "a" denotes the average value of the parameter enclosed in the circular parentheses. Figure 3(a)-(c) demonstrates the three patterns of distribution across the half thickness of sheet. When $D_{\mathrm{m}}=1$, the distribution is uniform. If $D_{\mathrm{m}}>1$ or $D_{\mathrm{m}}<1$, it means that there is a local bulge or indent with regard to the uniform distribution of $E / E_{\mathrm{tm}}$ within the region marked across the half thickness of sheet. Since Young's modulus $E$ is a constant, $D_{\mathrm{m}}>1$ implies the material within the local region suffers more voiding damage so that the value of $E_{\mathrm{tm}}$ is smaller than that in the major part of the distribution. Conversely, $D_{\mathrm{m}}<1$ indicates that voiding is locally alleviated and thus results in a larger value of $E_{\mathrm{im}}$.

In view of the variation of $E_{1 \mathrm{~m}}$ at different nodal points across half thickness, the finite-difference method is again applied to the present analysis to calculate the critical stresses $F_{i}$ for the cases of having inhomogeneity in voiding damage. The critical stress for the homogeneous case $\left(D_{\mathrm{m}}=1\right)$ is given as $F_{0}$. Figure 4(a) and (b) demonstrates the variation of the critical stresses with respect to varying the values of $D_{m}$. In the calculation, we take $E_{\mathrm{te}}=0$ and $\left(E / E_{\mathrm{tm}}\right)_{\alpha}=100$. The Poisson ratio and yield strain have the same values as given previously. It is obvious that bifurcations into either surface wrinkles or shear bands can be stabilized by the depression of voiding. Even a local alleviation (i.e. $D_{\mathrm{m}}<1$ ) can stiffen the materials to have critical stresses equal or slightly larger than the theoretical prediction for ideally homogeneous material (i.e. $D_{\mathrm{m}}=1$ ). When $D_{\mathrm{m}}<1$, the site of inhomogeneity has little influence on the critical stresses $F_{i}$.

On the other hand, catastrophic decrease in critical stress can happen if the situation is inverted when voiding is promoted locally (i.e. $D_{\mathrm{m}}>1$ ). Besides, if the promotion of voiding is at the site where the disturbance at bifurcation is largest, then the case becomes more devastating. Consequently, the pattern (a) of inhomogeneity distribution for surface wrinkling, but the pattern (c) for shear banding most impair the critical stresses. These are in accordance with the facts that the largest disturbance occurs at the top surface $\left(2 x_{3} / h=1\right)$ in surface wrinkling but moves to the middle plane $\left(2 x_{3} / h=0\right)$ in the case of shear banding, as shown in Fig. 2(a) and (b). The value of $h / L$ can be fixed as 1000 for shear banding, but varies as occasion requires to yield the lowest critical loading of each computed case in surface wrinkling.
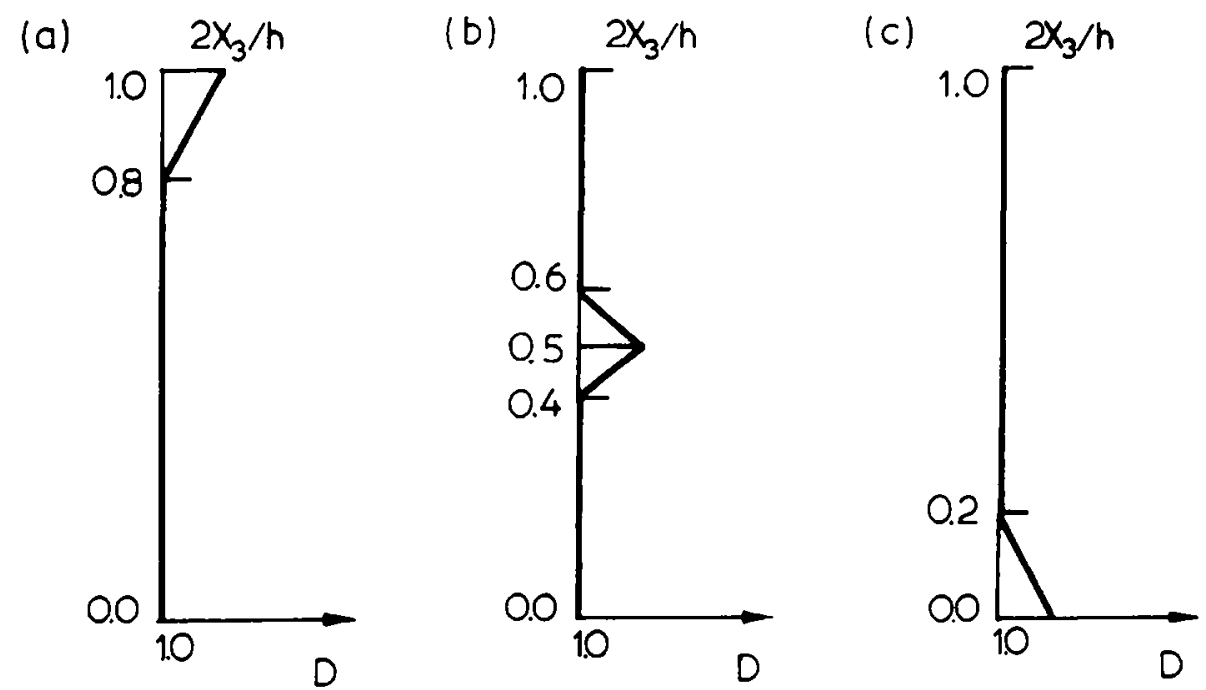

Fig. 3. Three patterns of distribution across the half thickness of sheet. 
Finally, the inhomogeneity of voiding damage plays a crucial role in varying the velocity disturbance at bifurcation. A demonstration of this effect is illustrated in Fig. 5, in which the velocity patterns $V_{2} /\left(V_{2}\right)_{\max }$ along the half thickness in Fig. 2(a) and (b) are now reproduced by using dotted lines for the homogeneous cases $\left(D_{\mathrm{m}}=1\right)$ of surface wrinkling and shear banding in Fig. 5(a) and (b), respectively. We use two extreme cases, the pattern (a) and pattern (c) in Fig. 3, to exemplify the inhomogeneity effects. In Fig. 5, the thick solid lines are based on the computations under the influence of pattern (a), while thin solid lines are depicted for those with pattern (c). Different degrees of inhomogeneity are taken in the examples as marked in Fig. 5.

It is obvious that the velocity disturbance at bifurcation becomes larger at the site where more voiding damage is suffered. Therefore, pattern (a) [thick lines] stimulates larger disturbances near
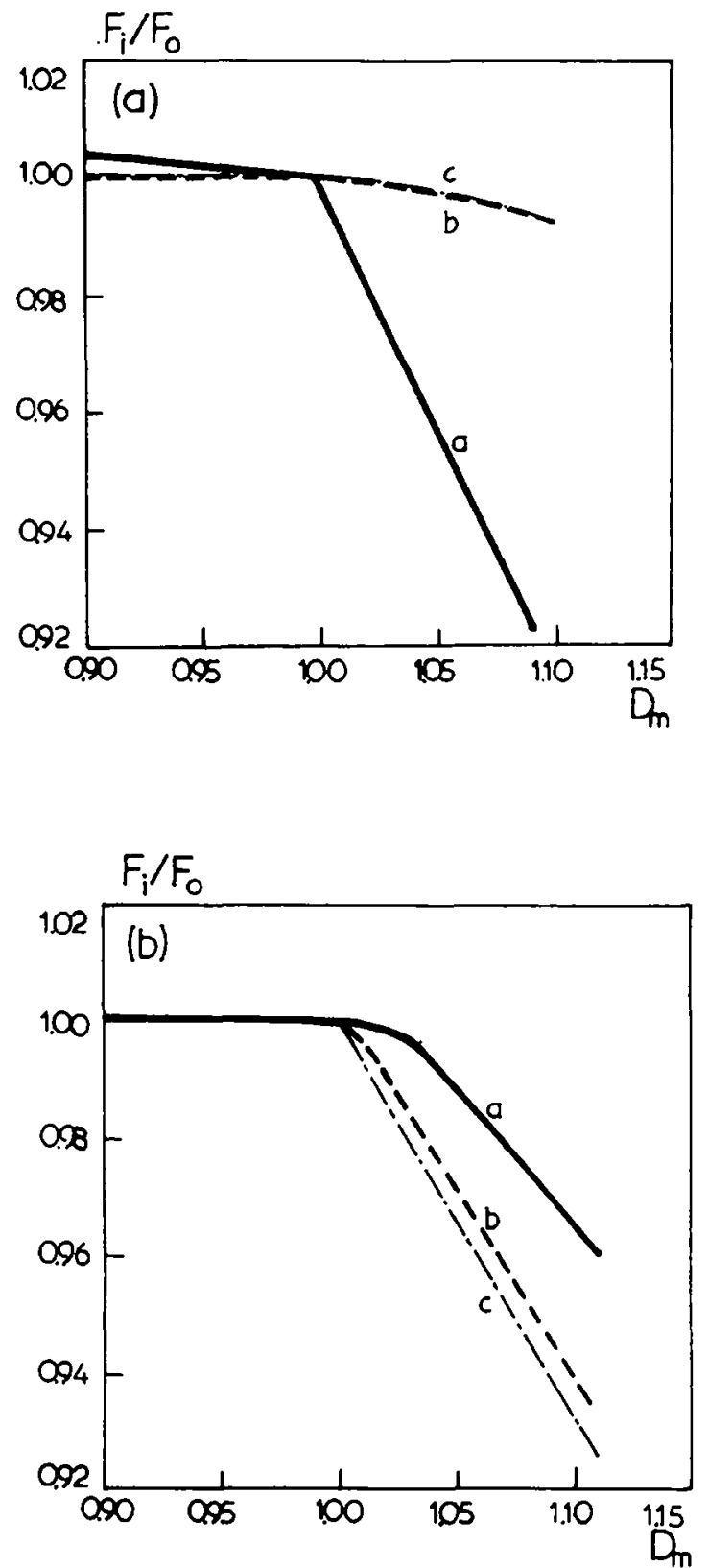

Fig. 4. The variation of the critical stress $F_{i}$ (normalized by $F_{0}$ ) with respect to varying the values of $D_{\mathrm{m}}$ $\left[E_{\mathrm{te}} / E=0,\left(E / E_{\mathrm{lm}}\right)_{\mathrm{a}}=100\right]$ (a) surface wrinkling, (b) shear banding. 

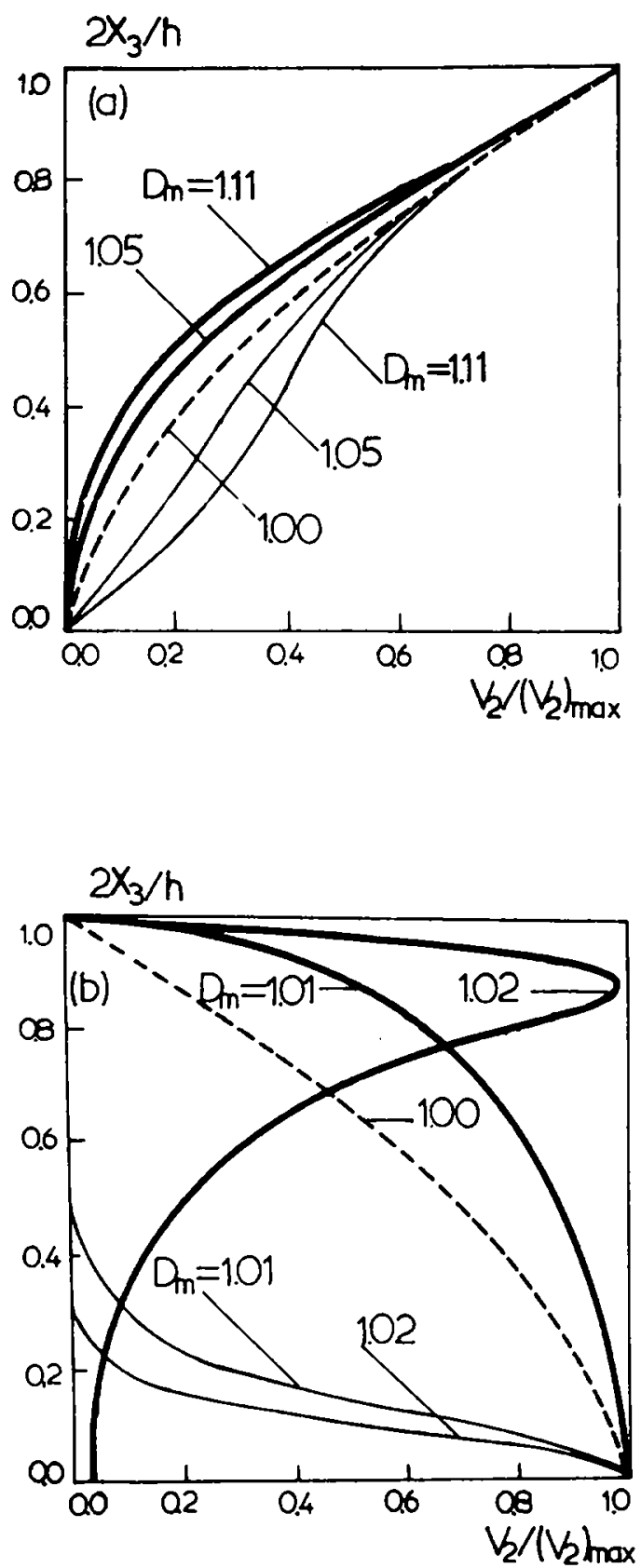

Fig. 5. The effect of inhomogeneity [with pattern (a) in thick solid lines and pattern (c) in thin solid lines] on the velocity disturbance at bifurcation (a) surface wrinkling, (b) shear banding.

to the surface $\left(2 x_{3} / h=1\right)$ while pattern (c) [thin lines] brings about more concentration near to the middle plane $\left(2 x_{3} / h=0\right)$. The violation degree of velocity disturbance increases with regard to the increasing of parameter $D_{\mathrm{m}}$. Shear banding is much more sensitive to the variation of $D_{\mathrm{m}}$, when compared with the situation of surface wrinkling.

\section{THE NONUNIFORMITY OF STRESS DISTRIBUTION}

When a sheet plate is subjected to uniaxial tension, the pre-bifurcation stress $\sigma_{11}$ may not be distributed uniformly along the thickness direction. In analysis, we have checked that if Poisson's ratio is adjusted appropriately within a range not larger than $5 \%$, the nonuniformity of stress $\sigma_{11}$ distribution across thickness would not bring about other stress components. Hence, the uniaxiality 
of stress state can be kept in a practical sense. Besides, it has also been checked that the variation in Poisson's ratio has a trivial effect on the critical values. Therefore, the process of previous calculation can be repeated for this case, except that the variation of uniaxial stress across the sheet thickness should be taken into account.

The patterns of nonuniformity distribution taken into consideration are analogous to those shown in Fig. 3. We let $\left(E / E_{\mathrm{tm}}\right)_{\mathrm{a}}$ and $\left(E / E_{\mathrm{tm}}\right)_{\mathrm{m}}$ be replaced by $\left(\sigma_{11}\right)_{\mathrm{a}}$ and $\left(\sigma_{11}\right)_{\mathrm{m}}$, respectively, and renew the definition for $D$ and $D_{\mathrm{m}}$ by taking

$$
D=\frac{\sigma_{11}}{\left(\sigma_{11}\right)_{\mathrm{a}}}, D_{\mathrm{m}}=\frac{\left(\sigma_{11}\right)_{\mathrm{m}}}{\left(\sigma_{11}\right)_{\mathrm{a}}}
$$

where the subscripts $\mathrm{m}$ and a have the same meaning as stated previously. $D_{\mathrm{m}}=1$ means uniform distribution of stress $\sigma_{11}$ along the sheet-thickness direction. $D_{\mathrm{m}}<1$ indicates that stress $\sigma_{11}$ is depressed locally, so the minimum value $\left(\sigma_{11}\right)_{\mathrm{m}}$ is smaller than the average value $\left(\sigma_{11}\right)_{\mathrm{a}}$. Conversely, if $D_{\mathrm{m}}>1$, it means that there is a local stress concentration, and thus the maximum value $\left(\sigma_{11}\right)_{\mathrm{m}}$ is larger than the average one.

For this case, let the voiding damage be homogeneous, the values assigned for the material parameters and the principle for determining the ratio of $h / L$ are the same as stated previously. The effects of nonuniformity in stress distribution on critical stresses are then calculated and delineated in Fig. 6. When $D_{\mathrm{m}}>1$, a similar trend as that illustrated in Fig. 4 can be seen. Local depression of stressing is stabilizing $\left(D_{\mathrm{m}}<1\right)$, but any flourish of stress amplitude $\left(D_{\mathrm{m}}>1\right)$ is not favourable for stabilization, especially if the stress peak is located at the place where the velocity disturbance should be large. Therefore, the most serious case for surface wrinkling is to have pattern (a) of nonuniform stress distribution across the thickness, since the maximum stress is at the surface plane $\left(x_{3}=h / 2\right)$ which is also the site of the largest velocity variation. Analogously, pattern (c) is most dangerous for shear banding, as the sensitive site moves to the middle plane at $x_{3}=0$. The effects of nonuniformity on the bifurcation patterns have a similar trend as that shown in Fig. 5. Larger velocity variation (than that of the uniform case) can be stimulated at the location of having larger stress.

\section{SLIGHT BIAXIALITY OF STRESS STATE}

As the geometric constraint to contraction in the transverse direction of sheet plate is increased, a tensile stress $\sigma_{22}$ is generated with increasing magnitude [17]. Slight biaxiality of stress state is inevitably existing in specimens loaded uniaxially.

Let us define

$$
\beta=\frac{\sigma_{22}}{\sigma_{11}}
$$

For convenience sake, the ratio value of $\beta$ is assumed to remain constant before material bifurcation. Taking into account this change of the pre-bifurcation stress state, we can determine the critical stresses by an analogous process as stated previously. In Table 2, the critical stresses are listed for shear banding under the condition of having $h / L=1000, v=0.3, E_{\mathrm{tc}} / E=0$, $E / E_{\mathrm{tm}}=100, \epsilon_{\mathrm{y}}=0.002$. It is obvious that the critical stress increases with increasing $\beta$, although the absolute value of $\beta$ is still small.

The critical stresses computed for surface wrinkling are plotted in solid lines against the values of $h / L$ with $\beta=0,0.02,0.05$ in Fig. 7. The general trends of the curves have some similarities with the results calculated on the basis of using Gurson's model [3] for constitutive relation and reported by Tvergaard [14]. Brief elucidation should be given for our distinctive points and findings. We use dotted lines to mark the critical stress listed in Table 2 for shear banding in the corresponding figures in Fig. 7. The gap between the dotted lines and solid curves increases with respect to the increase of $\beta$. This demonstrates that the shear banding in the plane is giving way to other bifurcation patterns as biaxiality increases. However, another type of shear banding crossing the transverse section of sheet can be favoured by biaxiality stress state, especially when it reaches a plane-strain pre-bifurcation case, e.g. the one shown by Tvergaard [14]. At the first sight of Fig. 7, it seems that necking $(h / L \rightarrow 0)$ is always leading in bifurcation, since its critical stress is always 

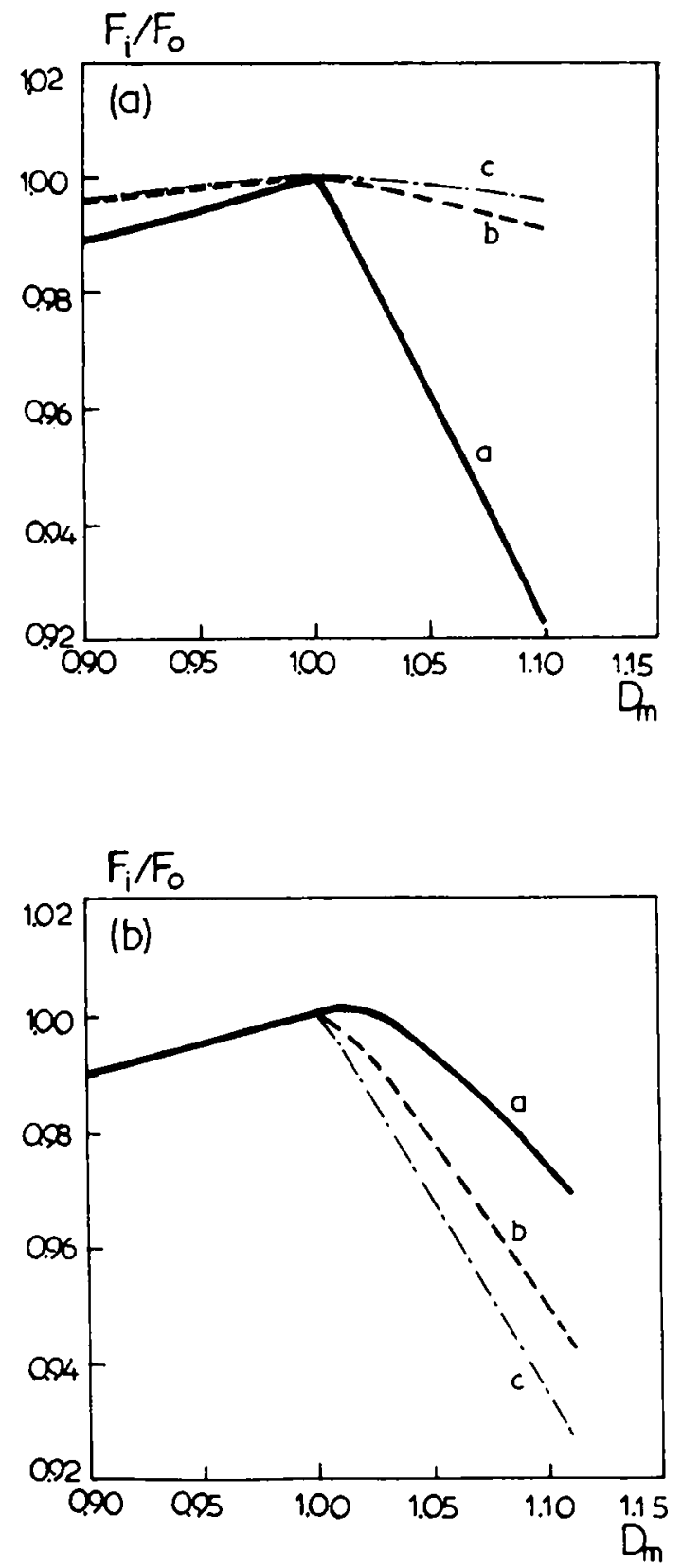

Fig. 6. The variation of the critical stress $F_{i}$ (normalized by $F_{0}$ ) with respect to varying the values of $D_{\mathrm{m}}$ $\left[E_{\mathrm{te}} / E=0,\left(E / E_{\mathrm{tm}}\right)_{\mathrm{e}}=100\right]$ (a) surface wrinkling, (b) shear banding.

lower than the others. But close examination on the corresponding patterns of bifurcation would show the complexity in this situation. If we examine a factor nominated as

$$
\gamma=\left(V_{2}\right)_{\max } /\left(V_{1}\right)_{\max },
$$

which is a ratio of maximum $V_{2}$ to maximum $V_{1}$ in which $V_{1}$ and $V_{2}$ have been defined in eq. (10). This ratio parameter is used to quantify the degree of transversal waviness vs the planar disturbance at bifurcation. Table 3 shows the change of parameter $\gamma$ with respect to $h / L$ and $\beta$. Although we have the lowest critical stress at $h / L=0$, the degree of waviness characterized by $\gamma$ is negligible,

Table 2. Critical stresses for shear banding under slight biaxiality of stress state $\left(h / L=1000, v=0.3, E_{k} / E=0\right.$,

\begin{tabular}{lllll}
\multicolumn{1}{c}{$\left.E / E_{\mathrm{tm}}=100, \epsilon_{\mathrm{y}}=0.002\right)$} \\
\hline$\beta$ & 0.0 & 0.02 & 0.03 & 0.05 \\
$\sigma_{\mathrm{c}} / \sigma_{\mathrm{y}}$ & 1.657 & 1.738 & 1.851 & 2.164 \\
\hline
\end{tabular}



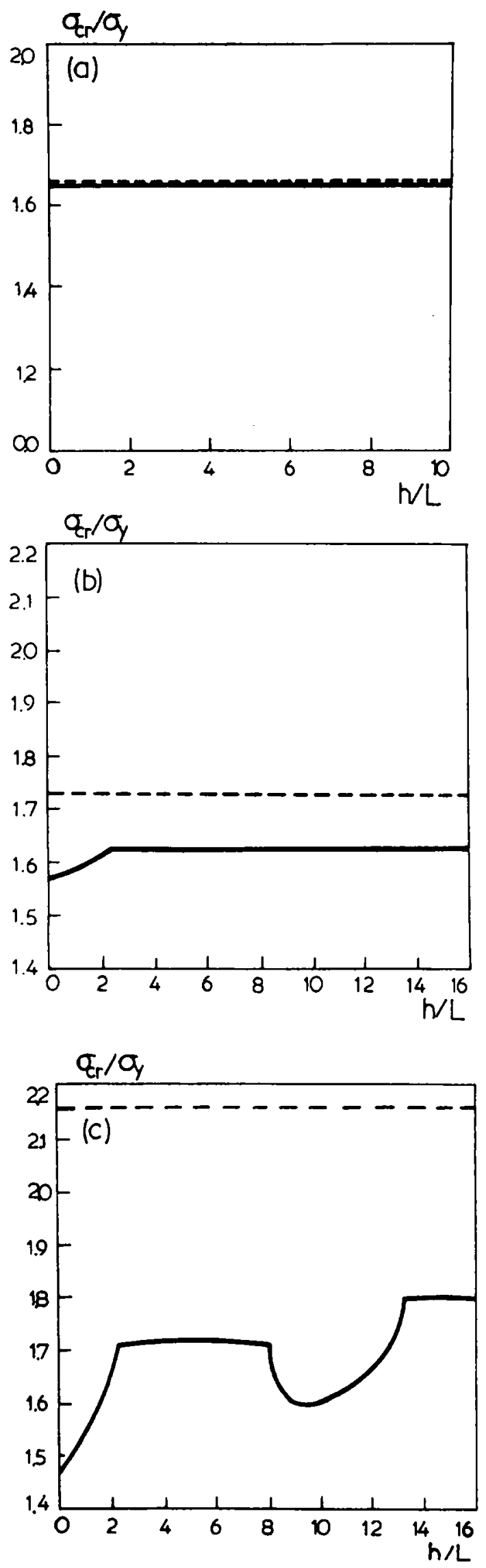

Fig. 7. Critical stress $\sigma_{s} / \sigma_{y}$ for surface wrinkling as function of parameter $h / L$ (a) $\beta=0$, (b) $\beta=0.02$, (c) $\beta=0.05$ 
Table 3. The parameter $;$ varying with respect to $h / L$ and $\beta\left(v=0.3, E_{\mathrm{t} /} / E=0, E / E_{\mathrm{lm}}=100, \epsilon_{\mathrm{y}}=0.002\right)$

\begin{tabular}{|c|c|c|c|c|c|c|c|c|}
\hline$\beta$ & $h / L$ & 0 & 0.5 & 1.0 & 1.5 & 2.5 & 3.0 & 3.6 \\
\hline 0 & & 0.0003 & 0.0009 & 0.027 & 0.036 & 0.041 & 0.049 & 0.053 \\
\hline 0.02 & & 0.003 & 0.008 & 0.07 & 0.09 & 0.12 & 0.13 & 0.14 \\
\hline 0.05 & & 0.005 & 0.009 & 0.15 & 0.18 & 0.23 & & \\
\hline
\end{tabular}

the disturbance is mainly restricted within the plane. We can have either necking $(h / L \ll 1)$ or surface wrinking $(h / L \gg 1)$ when the value of $\gamma$ becomes significant (e.g. if $h / L>3$ for $\beta=0.02$, or $h / L>1$ for $\beta=0.05$ ). It is obvious that necking with a small value of $h / L$ is more likely to occur when practical biaxiality is intensified.

\section{CONCLUSIONS}

Based on the numerical studies stated above, we can conclude:

1. Concerning the different patterns of material bifurcation, the numerical method can provide better ground for searching for the lowest critical loads, since numerical discretization offers more flexible representation to the distributions of velocity variations.

2. Generally speaking, the inhomogeneity of voiding damage and the nonuniformity of stress distribution bring about reduction in the critical loading. This explanation may unravel the puzzling question of having tested values of critical stress/strain lower than those predicted theoretically.

3. Depressing porosity development or alleviating stressing, even locally across the transversal section of sheet, can be beneficial for material stability. Therefore, developing surface treatment techniques to condition the internal state of sheet can be a practical implementation for the purpose of improving material ductility.

4. The critical stresses for surface wrinkling and shear banding have closely related values in plane sheet under uniaxial tension. This fact implies that materials at bifurcation can have multiple patterns. Furthermore, the influence of slight biaxiality state caused by geometric constraint on both the bifurcation pattern and critical stress enhances the perplexity of the problem.

\section{REFERENCES}

1. Hill, R. and Hutchinson. J. W., Bifurcation phenomena in the plane tension test. J. Mech. Phys. Solids 1975, 23, 239-264.

2. Li, G. C. and Zhu, C., Formation of shear bands in plane sheet. Int. J. Plasticity 1995, 11, 605-622.

3. Gurson, A. L., Yield criteria and flow rules for porous ductile media. J. Engng Mater. Technol. 1977, 99, 2-15.

4. Yamamoto, H., Conditions for shear localization in the ductile fracture of void-containing materials. Int. J. Fracture $1978,14,347-365$.

5. Ohno, N. and Hutchinson, J. W., Plastic flow localization due to nonuniform void distribution. J. Mech. Phys. Solids 1984, 32, 63-85.

6. Becker, R., The effect of porosity distribution on ductile failure. J. Mech. Phys. Solids 1987, 35, 577-599.

7. Huang, Y., The role of nonuniform particle distribution in plastic flow localization. Mechanics of Materials 1993, 16, 265-279.

8. McMeeking, R. M. and Rice, J. R., Finite element formulations for problems of large elastic-plastic deformation. Int . J. Solids Structures 1975, 11, 601-616.

9. Burke, M. A. and Nix, W. D., A numerical study of necking in plane tension test. Int. J. Solids Structures 1979, 15, 379-393.

10. Li, G. C., On dilatational plastic constitutive equation of ductile materials and plastic loading paths at bifurcation. Science in China (Ser. A) 1991, 34, 825-834.

11. Li, G. C., Liu, H. Q., Du, M. L., Hong, Y. S. and Zhang, X., Crack tip behaviour and crack propagation in ductile materials. Fatigue Fracture Engng Mater. Struct. 1992, 15, 187-202.

12. Liu, H. Q. and Li, G. C.. Plastic stress-strain fields and blunting effects during large deformations. Engng Fracture Mech. 1992, 42, 737-745.

13. Hutchinson, J. W. and Tvergaard, V., Surface instabilities on statically strained plastic solids. Int. J. Mech. Sci. 1980, 22, 339-354.

14. Tvergaard, V., Influence of void nucleation on ductile shear fracture at a free surface. J. Mech. Phys. Solids 1982, 30, $399-425$.

15. Li, G. C. and Zhang, Y. Z., Bifurcation and fracture in sheet-metal forming with void growth effect (in Chinese). Acta Mech. Sin. 1990, 22, 302-310.

16. Zhu, C., Hong, Y. S. and Li, G. C., Characteristics of shear banding in dual phase steel. Mater. Sci. Technol. 1993, 9, 1037-1043.

17. Chakrabarti, A. K. and Spretnak, J. W., Instability of plastic flow in the directions of pure shear: Il. Experimental. Metall. Trans. A 1975, 6A, 737-748. 